\title{
$150 \mathrm{keV}$ Emission from PKS2149-306 with BeppoSAX
}

\author{
Martin Elvis ${ }^{1}$, Fabrizio Fiore ${ }^{1,2,3}$, Aneta Siemiginowska ${ }^{1}$, Jill Bechtold ${ }^{4}$, Smita Mathur ${ }^{1}$ \& \\ Jonathan McDowell ${ }^{1}$ \\ ${ }^{1}$ Harvard-Smithsonian Center for Astrophysics, Cambridge MA 02138, USA \\ 2 Osservatorio Astronomico di Roma \\ via Osservatorio, Monteporzio-Catone (RM), I00040 Italy \\ 3 BeppoSAX Science Data Center \\ via Corcolle 19, Roma I00100 Italy \\ 4 Steward Observatory \\ Astrophysical Journal 544, 545 (2000)
}

\begin{abstract}
A BeppoSAX observation of the $\mathrm{z}=2.34$ quasar PKS2149-306 produced a strong signal in the high energy PDS instrument up to a maximum observed energy of nearly $50 \mathrm{keV}, 150 \mathrm{keV}$ in the quasar frame. The Beppo-SAX spectrum spans almost 3 decades $(0.3-150 \mathrm{keV}$, quasar frame) and shows an extremely hard $(\alpha=0.4 \pm 0.05)$ X-ray spectrum above $3 \mathrm{keV}$ (comparable to XJB the X-ray background slope), and either a softer $\left(\alpha=1.0_{-0.3}^{+0.6}\right)$ low energy component, or an ionized absorber at zero redshift. No evidence is seen of an Fe-K emission line $(\mathrm{EW}<167 \mathrm{eV}$ at $6.5 \mathrm{keV}$ quasar frame), a Compton hump $(R<0.3)$. A bremsstrahlung fit gives $\mathrm{kT}$ (rest) $=46_{-16}^{+32} \mathrm{keV}$, similar to the X-ray background value, and a high energy cut-off power law requires $\mathrm{E}_{\text {cut }}>120 \mathrm{keV}$ (quasar frame).

The SED of PKS $2149-306$ shows two peaks at $\sim 10^{12 \pm 0.5} \mathrm{~Hz}$ and $\sim 10^{21 \pm 1.0} \mathrm{~Hz}$ $(\sim 0.3 \mathrm{~mm}$ and $\sim 4 \mathrm{MeV})$, strongly resembling a low energy cutoff BL Lac object (LBL). The ratio of the two peaks shows an extreme Compton dominance $\left(C_{D}=1.4 \pm 0.4\right)$, as in flat spectrum radio quasars (FSRQs). The presence of an additional 'optical/UV big bump' component may provide photons that cool the jet, suppressing the radio emission.
\end{abstract}

Subject headings: quasars - emission mechanisms - X-rays - Gamma-rays 


\section{Introduction}

High redshift quasars are of interest not only for their 'record setting' quality, but also because they can tell us about the formation of quasars and about conditions in the universe at early times (Turner 1991, Hamann \& Ferland 1994). We should also be able to learn about the quasar emission processes by comparing the continua of quasars at low $\mathrm{z}$ with those of their cousins at the epoch at which the characteristic quasar luminosity peaks around $\mathrm{z} \approx 2$ (Boyle et al. 1987). Since small changes in physical conditions can greatly alter the tails of particle energy distributions the extreme high energy continuum may be a particularly good place to look for evolutionary effects.

So far though X-ray spectra of high redshift quasars from ROSAT (Elvis et al., 1994a, Fiore et al., 1998a) and ASCA (Elvis et al., 1994b, Cappi et al., 1997) have been few, and of limited signal-to-noise. Even so they have begun to show redshift dependent features that challenge models and require further XJB investigation: low energy cut-offs were discovered with ROSAT (Elvis et al., 1994a), and appear to be intrinsic (Fiore et al., 1998a, Elvis et al., 1998) and related to ultraviolet absorbing outflows; no Compton reflection component has been seen in z $>1$ quasars $\left(\Omega_{d} / 2 \pi \leq 0.4\right.$ [90\%], Elvis et al. 1994b, Nandra et al., 1995, Siebert et al., 1995, see also Williams et al (1992) for low z examples); nor is a $6.4 \mathrm{keV}$ (rest frame) iron line required by the fits $(\mathrm{EW}<20 \mathrm{eV}$ [90\%] Siebert et al. 1995; EW<120 eV (90\%) Elvis et al., 1994b). These properties differentiate high z quasars from typical low redshift Seyfert galaxies which have strong iron K-lines $(\mathrm{EW}=100-300 \mathrm{eV})$, and a strong Compton hump that requires half the sky (as seen from the X-ray source) to be covered with Compton-thick 'cold' material $\left(\Omega_{d} / 2 \pi=1\right.$, Nandra \& Pounds 1994 and references therein). This suggests a different structure within high luminosity quasars.

We observed PKS2149-306 as part of an AO1 GO program on BeppoSAX in order to examine these issues. At $\mathrm{z}=2$ the Compton hump region is well within the BeppoSAX (Boella et al., 1997a) 1.3-10 keV Medium Energy Concentrator Spectrometer (MECS, Boella et al., 1997b) band, making a measurement feasible. Moreover the good sensitivity of the high energy Phoswich Detector System (PDS, FWHM=1.4 degrees, Frontera et al., 1997) on BeppoSAX allows the extension of the spectrum to energies $(15-100 \mathrm{keV})$ where a Compton hump, bremsstrahlung and a power law can be more readily distinguished

PKS2149-306 is a $\mathrm{z}=2.34$ flat spectrum $\left(\alpha_{2.7}^{4.85 \mathrm{GHz}}=0.0 \pm 0.14\right.$, where $\left.f_{\nu} \propto \nu^{-\alpha}, \mathrm{NED}\right)$, core-dominated f radio-loud quasar with $m_{V}=17.9$. PKS 2149-306 is extraordinarily bright

1 The $2.3 \mathrm{Ghz}$ data of Morabito et al (1986) shows the source to be $>70 \%$ compact on a 3mas scale (24 pc at $\mathrm{z}=2.34, H_{0}=50 \mathrm{~km} \mathrm{~s}^{-1} \mathrm{Mpc}^{-1}, \Omega=1$ ), using the Parkes $2.7 \mathrm{Ghz}$ total flux (Quiniento \& Cersosimo 
in the ROSAT Sky Survey $\left(\mathrm{f}_{X}=10^{-11} \mathrm{erg} \mathrm{cm}^{-2} \mathrm{~s}^{-1}\right.$, Siebert et al., 1995, Brinkmann et al., 1995b, Schartel et al., 1996). Being bright, PKS2149-306 also promised to give the best possible limits on high energy emission from quasars. Instead, it gave us a clear detection. We present here the BeppoSAX 0.1-130 keV data for PKS2149-306, along with the broad band radio to $\gamma$-ray spectral energy distribution (SED) of the quasar, and we compare these with recent Blazar models.

\section{Observations and data analysis}

BeppoSAX observed PKS2149-306 from 1997 October 31 through 1997 November 1. The two imaging detectors - the 1.3-10 keV MECS and the 0.1-10 keV Low Energy Concentrator System (LECS, Parmar et al., 1997) - detected the quasar with high signal-to-noise at the center of the their field-of-view, at $0.105 \mathrm{ct} / \mathrm{s}$ and $0.05 \mathrm{ct} / \mathrm{s}$ respectively. (With exposures of 39,439 s and 17,862 s respectively.) Unexpectedly, the co-aligned Phoswich Detector System (PDS, Frontera et al., 1997) also detected a clear signal in its 15-100 keV bandpass of $0.26 \pm 0.05 \mathrm{ct} / \mathrm{s}$ (in an effective on-source exposure time of 16,676 s).

Standard data reduction was performed using the SAXDAS software package (1997). In particular, data are linearized and cleaned from Earth occultation periods and unwanted periods of high particle background (due to satellite passages through the South Atlantic Anomaly, SAA). Data from MECS units 2 and 3 were combined after gain equalization (unit 1 has been inoperative since 1997 May 7). The MECS and LECS pulse height spectra of PKS2149-306 were extracted using 3 arcmin and 8 arcmin radius extraction regions, respectively. These radii maximize the signal-to-noise ratio below $1 \mathrm{keV}$ in the LECS and above $2 \mathrm{keV}$ in the MECS. Background was taken from high Galactic latitude blank fields using the same extraction regions as for the source regions. Although background is negligible for this source, we checked that the level of the background in regions surrounding the source is within $10 \%$ of that recorded in the same regions in the blank fields.

For the PDS the problem of accurate background subtraction is crucial since the PDS is a non-imaging detector. The PDS uses rocking collimators, one for each of two pairs

of detectors, that switch positions quickly every 92 s so that one of the pairs is always pointed at the source, while the other is monitoring the background. The spectra from the four PDS crystals were summed together. The net source spectrum was then obtained by subtracting the 'off-source' background from the 'on-source' counts, scaling by the exposure time. The background in the equatorial BeppoSAX orbit varies by only $\sim 10-20 \%$, thanks to

1993) as a comparison. 
shielding by the Earth's particle belts (this was the main reason for selecting an equatorial orbit for BeppoSAX), and has an active anti-coincidence CsI(Na) particle shield (which is also used to detect gamma-ray bursts, Frontera et al., 1997). The main background variations are recorded just after the passages through the SAA. (The instrument is switched off while in the SAA). The first five minutes after SAA passages are excluded from the data analysis to allow an automatic gain control system to return the high voltages to their normal working value. This system keeps the pulse-height to energy scale constant to $1 \%$. Using the rocking technique it is possible to take into account accurately the residual background variations. The magnitude of residual systematic errors in PDS spectra have been evaluated

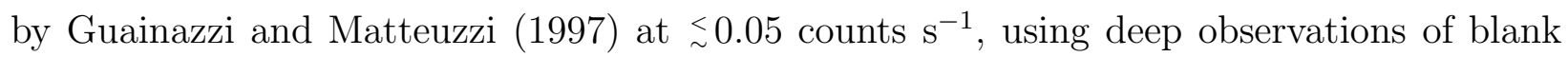
fields. Even if this maximum residual is subracted from the observed count rate, the signal to noise in this observation would remain $>4 \sigma$. The source is detected at $>3 \sigma$ up to 40-50 $\mathrm{keV}$, which is about $150 \mathrm{keV}$ in the quasar rest frame.

The chance of finding a source in any given 2 sq.degree, the FWHM beam area of the PDS, is small. The HEAO-1 A4 all sky catalog (Levine et al., 1984) lists just 7 high Galactic latitude $\left(|b|>20^{\circ}\right)$ sources in the $13-80 \mathrm{keV}$ band, which is closely comparable to the PDS band, down to a flux of $2 \times 10^{-10} \mathrm{erg} \mathrm{cm}^{-2} \mathrm{~s}^{-1}$ (10mCrab). The PKS2149-306 signal is 7.5 times fainter so, assuming a $\log \mathrm{N}-\log \mathrm{S}$ slope of -1.5 , we expect a chance coincidence rate of $1.4 \%$, hence we believe the PDS signal does come from PKS2149-306. Our confidence in the identification of the PDS signal with PKS2149-306 is increased further by the agreement to better than $10 \%$ of the normalizations of the PDS and MECS spectra and the similarity of their slopes (see next section). The EGRET source reported later is too far away to produce the PDS signal (see $\S 4$ ).

\section{X-ray Spectral Fitting}

Spectral fits were performed using the XSPEC 10.0 software package and versions of the response matrices made public on 1997 August 31. PI channels in MECS and LECS spectra are rebinned sampling the instrument resolution with the same number of channels at all energies and ensuring at least 20 counts per bin for the other. This guarantees the applicability of the $\chi^{2}$ method in determining the best fit parameters, since the distribution in each channel can be considered Gaussian.

Constant factors have been introduced in the model fitting in order to take into account the intercalibration systematics between instruments (BeppoSAX Cookbook, Fiore et al. 1998b). If we use the two MECS as reference instruments, then the LECS and PDS normalizations lie in the ranges $0.7-1.0$ and $0.80-0.95$ relative to the MECS, respectively. 
The (observed frame) energy ranges used for the fits are: $0.1-4 \mathrm{keV}$ for the LECS, 1.65$10.5 \mathrm{keV}$ for the MECS, and 14-130 keV for the PDS. All the results given in table 1 are in the observed frame. Quasar frame values are given in the text where appropriate. Errors in Table 1 are $90 \%$ confidence intervals for 1 interesting parameter. A simple power-law plus absorption model gives a reasonably good fit (Figure 1, Table 1), but with systematic deviations at low energies.

PKS2149-306 appears to have faded slightly over the three years between the obsevations: the 2-10 keV BeppoSAX flux, using the simple power law fit, is $(8.0 \pm 0.2) \times 10^{-12}$ erg $\mathrm{cm}^{-2} \mathrm{~s}^{-1}, 80 \%$ of the flux recorded in the 1994 October ASCA observation $(9.9 \times$ $10^{-12} \mathrm{erg} \mathrm{cm}^{-2} \mathrm{~s}^{-1}$, Cappi et al 1997); and the monochromatic $2 \mathrm{keV}$ BeppoSAX flux is $(2.8 \pm 0.1) \times 10^{-12} \mathrm{erg} \mathrm{cm}^{-2} \mathrm{~s}^{-1} \mathrm{keV}^{-1}, 76 \%$ of the $1990 / 1991$ ROSAT RASS detection at $(3.7 \pm 0.3) \times 10^{-12} \mathrm{erg} \mathrm{cm} \mathrm{cm}^{-2} \mathrm{~s}^{-1} \mathrm{keV}^{-1}$ (Schartel et al. 1996).

The LECS shows a clear excess of counts below about $1 \mathrm{keV}$. This soft excess seems in contradiction with the above Galactic $\mathrm{N}_{H}$ reported by Yaqoob et al. (1999). While variability in the 11 months (quasar frame) between the two observations is possible, we also note that the low energy calibration of ASCA is uncertain 9 , and we have more confidence in the Beppo-SAX value (Parmar et al., 1997, Mineo et al., 2000). The presence of a low energy excess means that the X-ray spectrum cannot give a reliable Galactic absorption value, so we fix the absorbing column to the Galactic $21 \mathrm{~cm}$ value $\left(2.2 \times 10^{20} \mathrm{~cm}^{-2}\right.$, Dickey \& Lockman 1990). The resulting energy spectral index, $\alpha=0.40 \pm 0.04$, is extremely flat (c.f. Sakano et al. 1998, Sambruna et al., 1999), although consistent with other high redshift radio-loud quasars measured with ASCA from 2-10 keV, including PKS2149-306 itself $\left(\alpha=0.42_{-0.02}^{+0.03}\right.$ Cappi et al. 1997, 0.54 \pm 0.05 Yaqoob et al. 1999). This slope is close to that of the X-ray background (Marshall et al., 1980) although this class of quasar is too rare to integrate to the X-ray background (see Elvis et al., 1994 and references. therein).

A better fit can be had with a broken power-law to account for the slight upturn in the LECS spectrum below $\sim 1 \mathrm{keV}$ (Table 1 ). In this case the steeper low energy slope is $2.0_{-1.0}^{+2.0}$ allowing the absorbing column to be free to vary, or $1.0_{-0.3}^{+0.6}$ fixing $N_{H}$ to its Galactic value. Both slopes are consistent with the ROSAT RASS value of $1.24 \pm 0.80$ (Schartel et al., 1996). The break energy is about $0.8 \mathrm{keV}$ (observed frame, $2.7 \mathrm{keV}$ quasar frame) in both cases. The high energy index is indistinguishable from the single power law index.

An apparent soft excess can also be generated though an absorption edge. In the quasar frame there is no sign of an ionized oxygen absorber, although at $z=2.34$ the energy of the

${ }^{2}$ see: http://heasarc.gsfc.nasa.gov/docs/asca/watchout.htm

http://heasarc.gsfc.nasa.gov/docs/asca/cal_probs.html 
PKS2149-306 BeppoSAX LECS, MECS, PDS

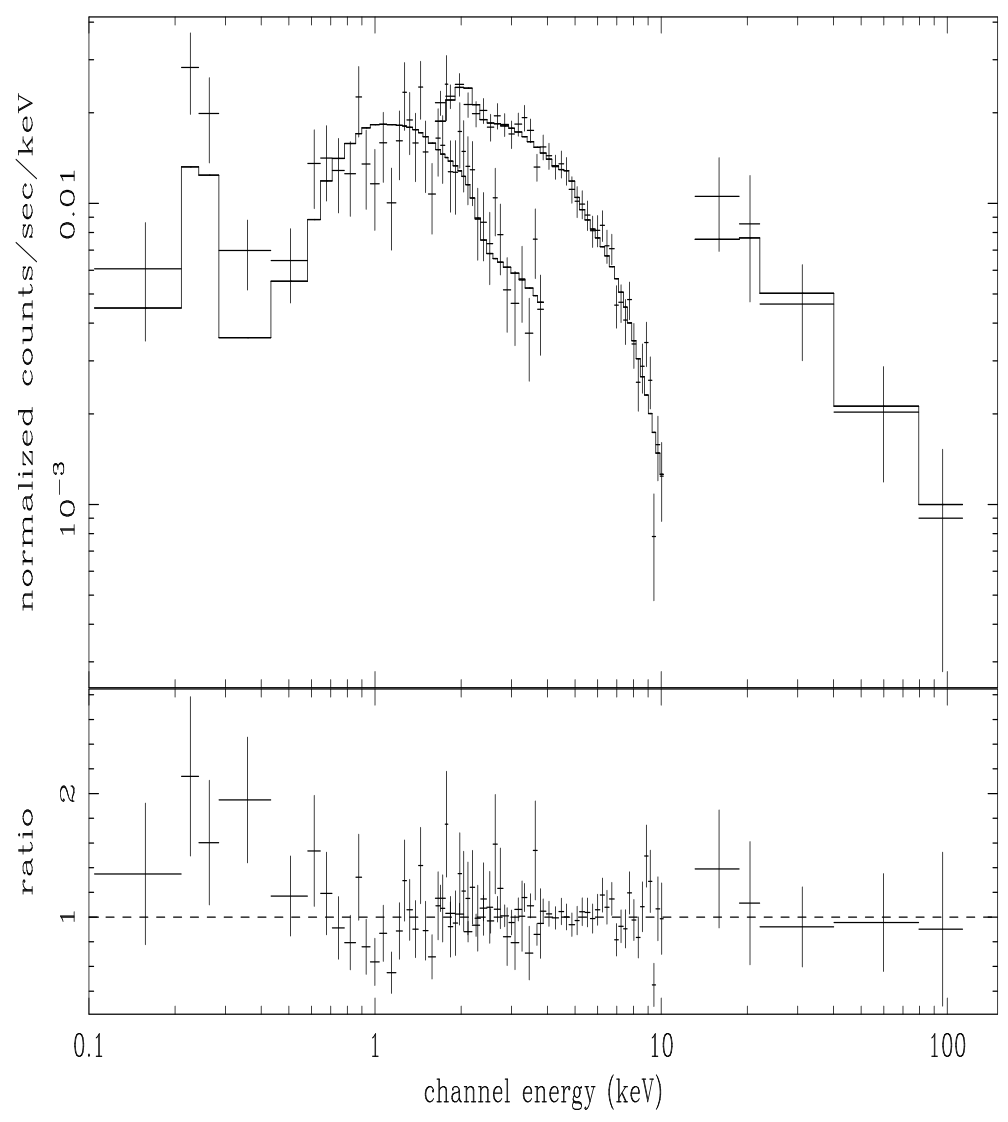

Fig. 1.- The 0.1-130 keV (observed frame) BeppoSAX LECS, MECS and PDS spectrum of PKS2149-306 fitted with a single power law model reduced at low energy by Galactic absorption. The lower panel shows the ratio between data and model. 
deepest edges usually seen in low luminosity Seyfert galaxies (OVII and OVIII) fall at 0.22 and $0.26 \mathrm{keV}$ (observer frame) respectively, close to the carbon edge $(0.283 \mathrm{keV})$ produced by the LECS window, and are therefore affected by large systematic errors. A fit with an absorption edge and a single power law (Table 1 ) gives a good $\chi^{2}$. The edge energy is $2.8 \mathrm{keV}$ in the quasar frame, which has no ready interpretation, but the observed energy of $0.8 \mathrm{keV}$ is consistent with either OVIII at $z<0.2$ or OVII at $z=0$. The optical depth is large, $\tau \sim 1$, implying a total hydrogen column of a few $10^{22} \mathrm{~cm}^{-2}$ assuming cosmic metal abundances. This is about one hundred times what it is seen from the Galactic $21 \mathrm{~cm}$ line. The high energy slope in this fit is consistent with the value from the broken power law fit.

No line is detected around $2 \mathrm{keV}$, the redshifted iron $\mathrm{K} \alpha$ energy. The $90 \%$ upper limits to the emission line equivalent width are $50 \mathrm{eV}$ and $25 \mathrm{eV}$ (observer's frame; $167 \mathrm{eV}$ and $84 \mathrm{eV}$ quasar frame) for the 6.4 and $6.7 \mathrm{keV}$ (quasar frame energies) iron $\mathrm{K} \alpha$ lines respectively. No line is seen at $5.1 \mathrm{keV}$ (observer's frame, $17 \mathrm{keV}$ quasar frame) with a limit of $63 \mathrm{eV}$ (90\% confidence, observer's frame), or $210 \mathrm{eV}$ (quasar frame). This compares with the $298_{-205}^{+202} \mathrm{eV}$ (quasar frame, $90 \%$ errors for two interesting parameters) measurement reported at thsi unusual energy by Yaqoob et al. (1999) from 1994 October ASCA data.

The presence of a steeper low energy component or absorption edge has little effect on the high energy slope. Since this steeper component is only important in the LECS data we shall fit only the MECS and PDS data when investigating the high energy component, as we do in the rest of this section. This has the advantage of removing extra free parameters from the models, which usefully decreases the allowed parameter space.

To determine the overall 5-150 keV rest frame spectral shape we next fitted three XSPEC models: a power-law with an exponential cutoff (cutoffpl); a Compton reflection model for neutral reflectors (pexrav, Magdziarz \& Zdziarski 1995); and a thermal bremsstrahlung model. In pexrav the redshift was frozen at the quasar redshift, the disk inclination was frozen at $\cos i=0.45$, and the cosmic abundances of Anders \& Ebihara (1982) were assumed. For both models the local absorption was fixed at the Galactic value.

The Compton reflection model (Table 1) gave a 90\% upper limit of 0.3 to the relative normalization, $R$, of the reflected component 5 . This compares with typical values of 1.0 in Seyfert galaxies (Matt 1998). The cut-off power-law model (Table 1) requires the cut-off energy, $\mathrm{E}_{c}$, to be $>36 \mathrm{keV}$ (90\% confidence, observed frame), or $>120 \mathrm{keV}$ in the quasar frame. A bremsstrahlung fit gives a rest frame temperature of $46_{-16}^{+32} \mathrm{keV}$ (90\% confidence, 1 interesting parameter), similar to that found in several $\mathrm{z}=3$ radio-loud quasars in the 2$10 \mathrm{keV}$ band with ASCA (Cappi et al., 1998). The Beppo-SAX result suggests that these

\footnotetext{
${ }^{3} R=\Omega / 2 \pi$, for an isotropic source above a flat infinite disk
} 
temperatures are not merely an artifact of the upper energy limit of ASCA. As for ASCA, a bremsstrahlung model is a slightly worse fit than power-law models.

Table 1: Spectral fits (observed frame)

\begin{tabular}{|c|c|c|c|c|c|}
\hline model & $N_{H}^{a}$ & $\alpha_{H}$ & $\alpha_{S}$ & $\begin{array}{l}\text { additional } \\
\text { parameters }\end{array}$ & $\chi^{2}(\mathrm{dof})$ \\
\hline \multicolumn{6}{|c|}{ LECS, MECS, PDS fits } \\
\hline Power law & 2.2 FIXED & $0.4 \pm 0.04$ & - & - & $92.3(91)$ \\
\hline Broken p.l. & $5.0_{-2.8}^{+5.0}$ & $0.39 \pm 0.05$ & $2.0_{-1.0}^{+2.0}$ & $E_{b}=0.8 \pm 0.4$ & $81.2(88)$ \\
\hline Broken p.l. & 2.2 FIXED & $0.37 \pm 0.04$ & $1.0_{-0.3}^{+0.6}$ & $E_{b}=0.8 \pm 0.4$ & $84.1(89)$ \\
\hline P. L. + edge & 2.2 FIXED & $0.49 \pm 0.08$ & - & $\begin{array}{c}E_{e}=0.86 \pm 0.12 \\
\tau=0.9 \pm 0.5\end{array}$ & $80.9(89)$ \\
\hline \multicolumn{6}{|l|}{ MECS, PDS fits } \\
\hline Power law & 2.2 FIXED & $0.41 \pm 0.05$ & - & - & $46.9(51)$ \\
\hline Cut-off p.l. & 2.2 FIXED & $0.41 \pm 0.05$ & - & $E_{c}>36$ & $46.9(50)$ \\
\hline Compton refl. & 2.2 FIXED & $0.41 \pm 0.05$ & - & $R<0.3$ & $46.9(50)$ \\
\hline Bremsstrahlung & 2.2 FIXED & $\mathrm{T}^{b}=46_{-16}^{+32}$ & - & - & $54.9(51)$ \\
\hline
\end{tabular}

\section{The Spectral Energy Distribution of PKS2149-306}

The addition of the broad band BeppoSAX spectrum from 0.1-50 keV (observed) allows the construction of a well-sampled Spectral Energy Distribution (SED) since PKS2149-306 is already XJB well-observed at radio wavelengths, as shown by a NED 1 search. Additional radio (Quiniento et al., 1993) and millimeter data (Steppe et al., 1988) fill out the long wavelength SED. Although PKS2149-306 has a flat radio spectrum, Cersosimo et al (1994) include PKS2149-306 as a candidate GigaHertz Peaked Spectrum (GPS) radio source, because of a mild curvature in its radio spectrum. PKS2149-306 also has low radio polarization $(<1.7 \%$ at $1.4 \mathrm{MHz}$, Condon et al 1998). These two characteristics of GPS sources make it unlikely that such sources are dominated by beamed emission (O'Dea 1998). PKS 2149-306 though, has a Blazar-like SED and is unlikely to be a normal GPS source. The curvature in the PKS2149-306 radio spectrum may instead be due to the Compton downshifting of the originating electron spectrum, caused by a quenching UV source (see $\$ 5.1$ )

\footnotetext{
${ }^{4}$ The NASA/IPAC Extragalactic Database (NED) is operated by the Jet Propulsion Laboratory, California Institute of Technology, under contract with the National Aeronautics and Space Administration.
} 
In the $100 \mu \mathrm{m}$ to $0.1 \mu \mathrm{m}$ range there are only IRAS Faint Source Survey (FSS, Moshir et al., 1989) upper limits and an optical spectrum (Wilkes et al., 1983, Wilkes 1997, private communication). These few data points though are importantly constraining. A search of the IRAS FSS noise plates gives $3 \sigma$ upper limits of $130 \mathrm{mJy}, 96 \mathrm{mJy}, 70 \mathrm{mJy}$ and $600 \mathrm{mJy}$ at $12 \mu \mathrm{m}, 25 \mu \mathrm{m}, 60 \mu \mathrm{m}$ and $100 \mu \mathrm{m}$ respectively. These limits are stable against small changes in position, so that a complex background (e.g. from Galactic cirrus) is not causing spurious values.

The Compton Gamma Ray Observatory EGRET summed phase 1-4 (1991-1995) exposure $\left(9 \times 10^{8}\right.$ seconds) of the field shows no evidence for emission from PKS2149-306. A point source fit at the position of the quasar gives a 3 sigma upper limit of $6.9 \times 10^{-8}$ photons $\mathrm{cm}^{-2} \mathrm{~s}^{-1}(30 \mathrm{MeV}-3 \mathrm{GeV})$. We do recover the well known BL Lac object PKS 2155-304 (3EG J2158-3023, Hartman et al 2000) with a flux of $(1.2 \pm 0.3) \times 10^{-7}$ photons cm ${ }^{-2} \mathrm{~s}^{-1}$ (30 MeV-3 GeV). However, this source lies $1.7 \mathrm{deg}$ from PKS2149-306, well outside the 99 percent confidence region of the EGRET source position. The PDS reaches a transmission of 50 percent at 0.7 degrees and is effectively at zero transmission by 1.7 degrees, so PKS 2155-304 cannot be the origin of the PDS signal.

The complete SED of PKS2149-306 (Figure 2) shows how unusually hard and energetically dominant the hard X-ray, PDS, spectrum is when compared with normal radio-loud quasar SEDs (dot-dash curve, Elvis et al., 1994c). The whole Beppo-SAX spectrum lies an order of magnitude above the normal level for radio-loud quasars. The X-ray 'soft excess' ( $\mathrm{E}<3 \mathrm{keV}$ in the quasar frame) appears to be a smooth continuation of the $\alpha \sim 1.0$ optical slope (with the caveat that an ionized absorber could fake an excess, see §3). In contrast the 3-150 keV slope is harder by $\Delta \alpha \sim 0.5$. However, unless the source varies by a factor greater than 50 at $\sim 1 \mathrm{GeV}$, this hard X-ray MECS/PDS slope cannot extend up to EGRET energies, and must instead turn down somewhere between $120 \mathrm{keV}$ (the quasar frame lower limit on a cut-off in the PDS spectrum) and $40 \mathrm{MeV}$.

The SED of PKS 2149-306 (figure 2) seems to have two peaks at about $10^{12}$ and $10^{21}$ Hz. This 'two peaks' SED shape is similar to those of blazars (Fossati et al. 1998), strongly suggesting that the observed SED, outside the optical band, is dominated by a blazar continuum originating in a jet. The solid and dashed lines on the SED (figure 2) are spline interpolations of the highest frequency radio point and the X-ray data above $2 \mathrm{keV}$ constrained not to exceed the IRAS and EGRET upper limits. The solid line is a spline fit close to one of the synchro-Compton models from Ghisellini et al.(1998). The lines are chosen to indicate the uncertainty in determining the ratio of the two peaks. (The lines are not fits to the data with a physical model, and are only useful to guide the eye.) The two spline curves define the ranges of the two peak frequencies and amplitudes. The $\gamma$-ray peak lies at a few 


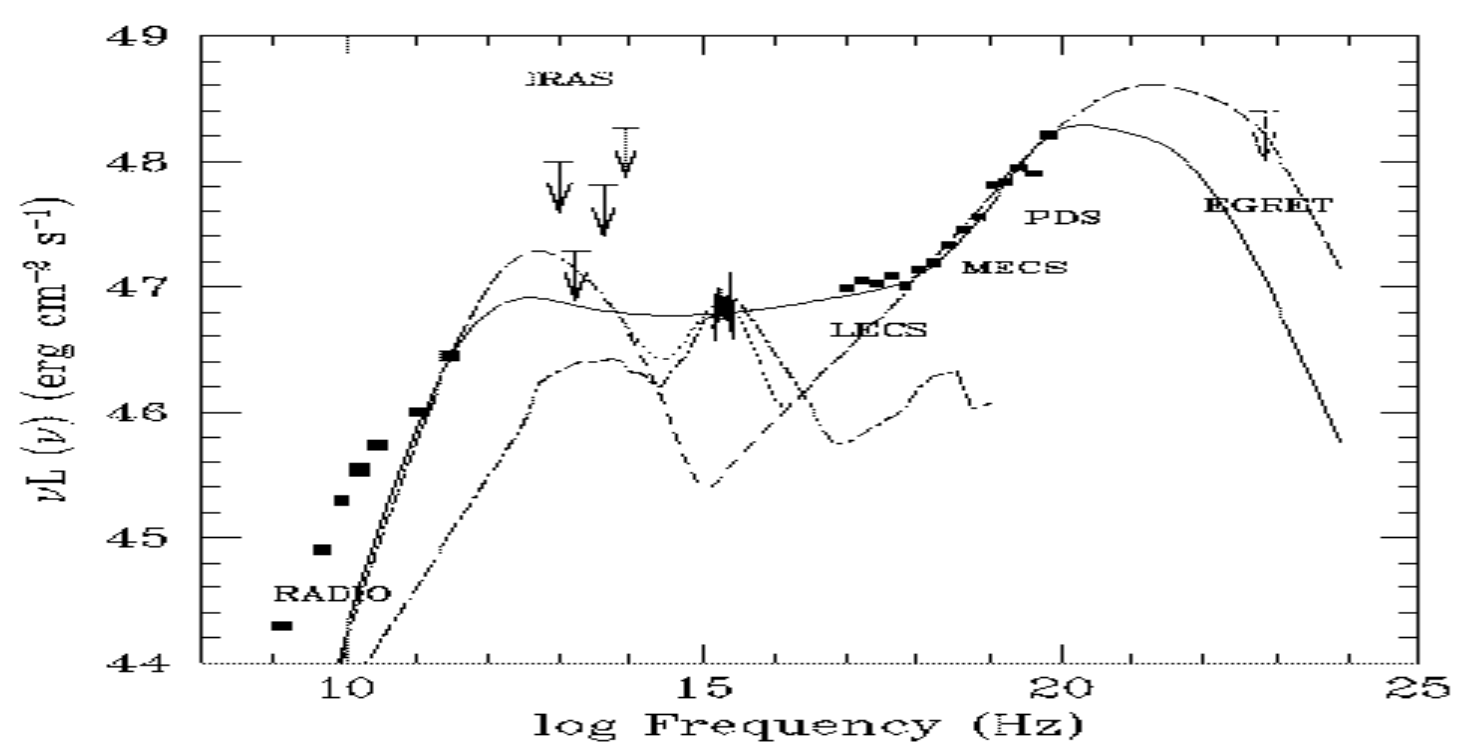

Fig. 2.- The radio to $\gamma$-ray SED of PKS2149-306. The solid line is a spline fit close to one of the synchro-compton models from Ghisellini et al.(1998). The long dashed line is a spline interpolation through the highest frequency radio point and the X-ray data that does not exceed the IRAS and EGRET upper limits. The two spline curves (solid and long-dash) illustrate the uncertainty in defining $C_{D}\left(=\log \left(L_{I C} / L_{\text {synch }}\right)\right.$. The thin dot-dash line is the mean SED of radio-loud quasars from Elvis et al. (1994c), normalized to the optical, to show how PKS2149-306 is a factor $\sim 10$ more X-ray loud than is typical. The short-dash line shows an illustrative accretion disk model (for $M_{b h}=10^{10} M_{\odot}, \dot{m} / m_{E d d}=0.1$ ) added to the long-dash curve, showing the likely large UV bump in PKS2149-306. 
$\mathrm{MeV}, \nu_{\text {peak }}(I C)=10^{21.0 \pm 1.0} \mathrm{~Hz}$, given the EGRET upper limit. The peak of the low frequency, radio- $\mathrm{mm}$, peak is in the sub-millimeter range, $\nu_{\text {peak }}($ synch $)=10^{12.0 \pm 0.5} \mathrm{~Hz}$. These are both unusually low frequencies. The ratio of the two peak luminosities, the Compton dominance, $C_{D}\left(=\log L_{I C} / L_{\text {synch }}\right.$, Ghisellini et al., 1998$)=1.4 \pm 0.4$, is near the top of the known range.

\section{Discussion}

\subsection{PKS 2149-306: An Extreme Compton Dominant Quasar}

The detection of hard X-ray emission in PKS 2149-306 reaches an energy some 4 times higher than detected in any other $\mathrm{z}>1$ quasar, except for the EGRET blazars. Moreover the X-ray to optical ratio of PKS 2149-306 is large, a factor 10 larger than normal for radio-loud quasars. Expressed as $\alpha_{O X}$, the optical $(2500 \AA)$ to X-ray (2 keV) slope, 0.81 , is unusually small. Only two radio-loud quasars out of some 250 in the survey by Brinkmann et al. (1995a) have $\alpha_{O X}<0.9$. Clearly there is something unusual about this quasar. The SED of PKS 2149-306 is not that of a typical, unbeamed, AGN. The 'two peaks' SED shape has not been seen in 'normal' unbeamed quasars (Elvis et al., 1994, Mattox 1994), although existing limits are surprisingly weak), but is common in relativistically beamed objects, both BL Lacs and quasars (Padovani \& Giommi 1996). The absence of reflection components (Compton hump and iron line) strongly limits the contribution to the observed flux of any Seyfert-like component, although these features are also weak in radio-quiet high luminosity quasars (Iwasawa \& Taniguchi 1993).

The 'two peaks' SEDs of BL Lac objects have often been considered within the SynchrotronInverse Compton formalism (e.g. Sambruna, Maraschi \& Urry 1996). In this scheme the low frequency (radio-mm) peak is the primary synchrotron peak, while the high frequency $(\gamma$ ray) peak comes from photons Inverse Compton scattered off the relativistic electrons. These scattered photons may be the synchrotron photons themselves (the self-Compton case), or from an external radiation source.

The frequencies at which the two components peak in $\log \nu f_{\nu} v s . \log \nu$ space varies by 5 decades, which changes their observational appearance greatly. Fossati et al. (1998) and Ghisellini et al. (1998) have suggested that the Blazars form a single, smooth sequence with bolometric luminosity, from the lower luminosity High energy cut-off BL Lacs (HBL) to the Low energy cut-off BL Lacs (LBL) and then to the high luminosity flat spectrum radio quasars (FSRQs, Sambruna, Maraschi \& Urry 1996, Padovani, Giommi \& Fiore 1997). Unlike HBLs and LBLs, FSRQs show normal quasar emission in the optical/UV, i.e. both the broad emission lines and the 'big bump' blue continuum (with a presumed accretion disk 
origin) are seen. This optical emission can occasionally dominate, as it does in PKS2149-306. We conclude that PKS2149-306 is a high luminosity FSRQ.

In PKS2149-306 both SED peaks lie at the extreme lower ends of the distributions in the Fossati et al (1998) and Ghisellini et al (1998) samples. In the Fossati et al. scheme the 'Compton dominance' (the ratio between the luminosity in the two peaks) is inversely proportional to the frequency of the synchrotron peak, roughly to the one half power (Figure 3). PKS2149-306 fits this correlation, in that the large Compton dominance of PKS2149-306 agrees with low frequency position of the synchrotron peak of its SED.

Ghisellini et al. go on to suggest that a strong external radiation source, such as an accretion disk powered 'optical/UV big bump', provides a bath of cool photons that can efficiently drain energy from jet electrons by Compton scattering them to high energies. The low energy photons may come directly from the disk below (Dermer \& Schlickeiser 1993) or from above or from the side (Siemiginowska \& Elvis 1994, Sikora et al., 1996). This cooling makes inverse Compton losses dominate over synchrotron losses making the radio spectrum weak relative to the X-ray, so creating a 'Compton dominant' SED. PKS2149-306 seems to have a large 'optical/UV big bump', as expected in this model. Such large optical/UV big bumps are unusual, but not unprecedented in FSRQ (e.g. 3C273, Elvis et al., 1994c). An illustrative accretion disk spectrum, that roughly fits the big bump, is shown in figure 2 $\left(M=10^{10} M_{\odot}, \dot{m} / \dot{m}_{E d d}=0.1\right.$, from the tabulation given in Siemiginowska et al. 1995).

\subsection{Low Energy Excess or Ionized Absorber?}

The soft X-ray excess in PKS 2149-306 appears unusually strong (Figure 2). We noted in $\$$ 国 that an absorption edge imprinted onto a single power law gives an equally good fit. Since ionized absorbers of the kind that could produce such an edge are common in AGN and blazars we must consider this interpretation on an equal footing with a 'soft excess' component.

An absorption edge can be more tightly constrained than a soft excess. A local z 0 absorber is unlikely since no molecular cloud is nearby (Marscher 1988, Turner et al., 1995), nor is any low z galaxy seen on the STScI digitization of the sky surveys. An absorber associated with the quasar is more likely, X-ray absorption with a similar column density is common in radio-loud high redshift quasars (Elvis et al., 1994a, Cappi et al., 1997, Fiore et al., 1998a, Elvis et al., 1998), and there is evidence for high ionization oxygen absorbers of similar column density in some low redshift blazars (Canizares \& Kruper 1984, Koenigl et al. 1995, Sambruna et al. 1997, Grandi et al. 1997, Cagnoni \& Fruscione 2000), though 


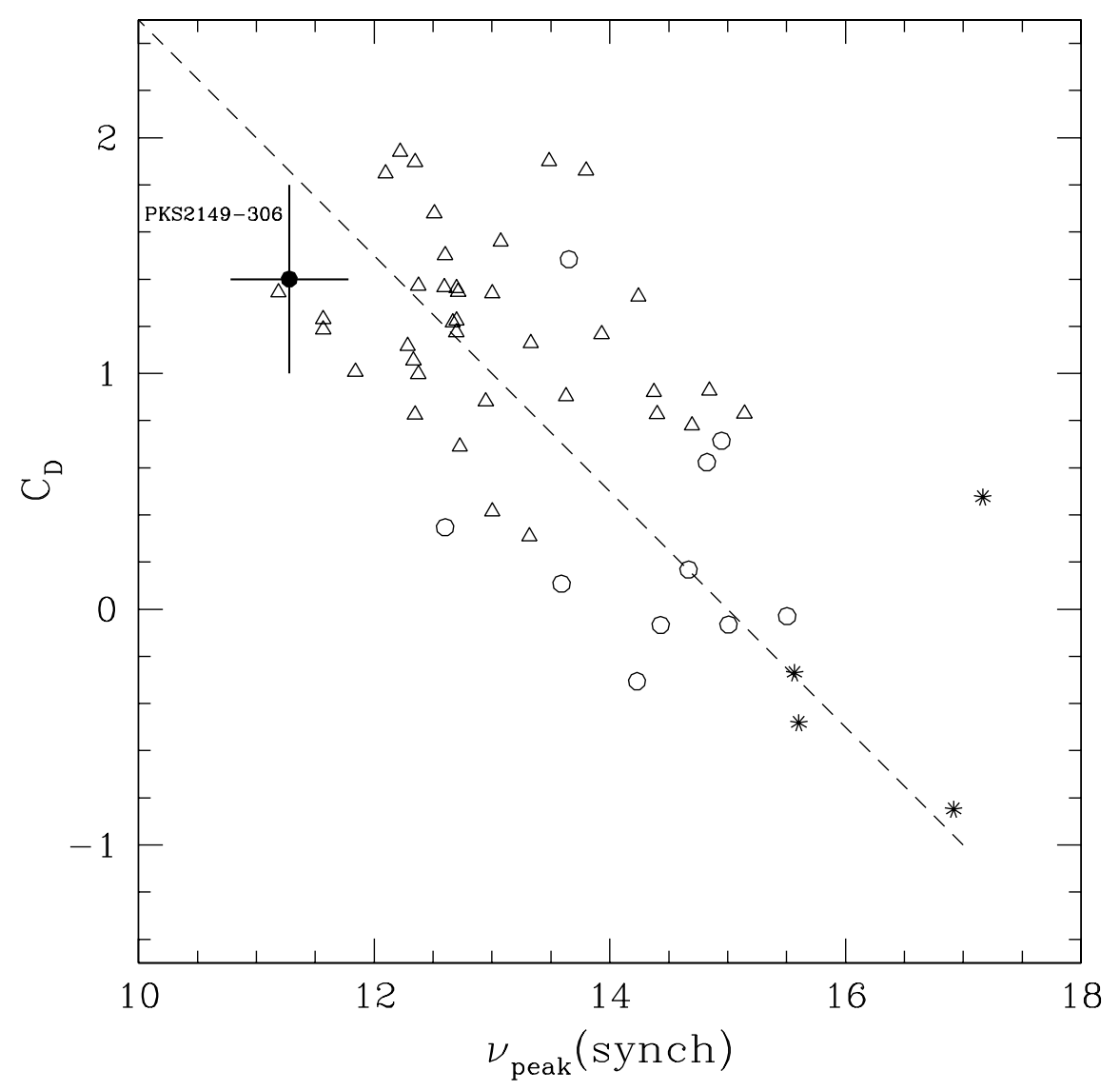

Fig. 3.- Compton dominance $\left(C_{D}=\log \left(L_{I C} / L_{\text {synch }}\right)\right.$ vs. observed synchrotron peak XJB frequency $\left(\nu_{\text {peak }}(\right.$ synch $)$, after Ghisellini et al., 1998). PKS2149-306 is the solid point with the error bars. A $C_{D} \propto \nu_{\text {peak }}(\text { synch })^{-0.5}$ relation is shown. Stars are HBLs (High energy cutoff BL Lacs); open circles are LBLs (Low energy cutoff BL Lacs); triangles are FSRQs (Flat Specrum Radio Quasars). 
these absorbers are not always seen (Chiappetti et al. 1999). Chandra high resolution grating spectra however do show comparable features (Fruscione et al. 2000, in preparation). To produce an apparent $z=0$ absorber would require a coincidence of outflow velocity and cosmological redshift. A substantial ejection velocity, $v=0.6 c$, is implied. In PKS 2155-304 the absorber has a suggested velocity of $\Delta v \sim 0.1 c$ relative to the core (Canizares \& Kruper 1984) so a high ejection velocity in PKS 2149-306 is not inconceivable. $v=0.6 c$ is larger than the $v=0.1-0.2 c$ seen in broad absorption line quasars (Turnshek 1988); it is much slower, though, than the velocities needed to explain superluminal radio sources (Zenzus 1997), although these may be pattern speeds rather than true expansion velocities. Only X-ray spectra with significantly better resolution can definitively identify absorption edges. Chandra grating observations are scheduled.

\section{Conclusions}

We have detected the $\mathrm{z}=2.34$ quasar PKS2149-306 up to $150 \mathrm{keV}$ in its rest frame, using the PDS on BeppoSAX. This is some 4 times higher in energy than any other $\mathrm{z}>1$ quasar, except for the EGRET blazars. PKS 2149-306 is also an order of magnitude X-ray loud relative to the optical $\left(\alpha_{O X}=0.81\right)$. The $>3 \mathrm{keV}$ spectrum of PKS 2149-306, as for several other high redshift radio-loud quasars, is extremely hard $\left(\alpha=0.41 \pm 0.05, \mathrm{kT}=46_{16}^{32} \mathrm{keV}\right.$, observed frame), comparable to the X-ray background spectrum.

The PKS2149-306 SED seems to show the two peaks typical of BL Lacertae objects, suggesting that the X-ray 1-100 keV observed emission is dominated by an inverse Compton component. Unusually the SED is strongly dominated by the $\gamma$-ray peak. A strong 'big bump' in the optical/ultraviolet seems to be present in PKS2149-306. Possibly, as suggested by Ghisellini et al. (1998), this bump is the source of the photons that cool the jet, suppressing the radio emission and creating the Compton dominated spectrum. This makes PKS2149-306 an extreme example of the luminosity based one-parameter unification scheme of Ghisellini et al..

The low energy X-ray spectrum of PKS 2149-306 shows no absorption above the Galactic value, contrary to the ASCA 1994 data (Yaqoob et al. 1999), showing instead a soft excess. An absorption edge fits the data as well as a broken power-law. One interpretation

of an absorption edge is an oxygen absorber with $\mathrm{N}_{H} \sim 10^{22} \mathrm{~cm}^{-2}$, blueshifted from the quasar redshift and implying an ejection velocity, $v \sim 0.6 c$. Such an absorber would provide a link with absorbers in other high redshift quasars, and with the absorbers in some low z blazars. However, the data are ambiguous and higher spectral resolution observations are needed to check this result. 
The Beppo-SAX data set strong limits on the X-ray reflection features: $\mathrm{EW}(\mathrm{Fe}-\mathrm{K})<15 \mathrm{eV}$ (quasar frame) and $R<0.3$ for the Compton Hump.

Hard X-ray observations, as we hoped when initiating this project, do seem to select physically extreme objects.

We thank Belinda Wilkes for providing a digital version of her optical spectrum of the quasar, Daryl Macomb for providing the EGRET upper limit, Seth Digel for the IRAS data, Gabriele Ghisselini for the data points in figure 3, and the referee for a careful reading that improved the paper. This work was supported in part by NASA contract NAS8-39073 (ASC), and NASA grants NAGW-2201 (LTSA) and NAGW- (ADP). This research has made use of data obtained through the High Energy Astrophysics Science Archive Research Center Online Service, provided by the NASA-Goddard Space Flight Center. This research has made use of the NASA/IPAC Extragalactic Database (NED) which is operated by the Jet Propulsion Laboratory, Caltech, under contract with the National Aeronautics and Space Administration Based on photographic data obtained using The UK Schmidt Telescope. The UK Schmidt Telescope was operated by the Royal Observatory Edinburgh, with funding from the UK Science and Engineering Research Council, until 1988 June, and thereafter by the Anglo-Australian Observatory. Original plate material is copyright (c) the Royal Observatory Edinburgh and the Anglo-Australian Observatory. The plates were processed into the present compressed digital form with their permission. The Digitized Sky Survey was produced at the Space Telescope Science Institute under US Government grant NAG $\mathrm{W}-2166$.

\section{REFERENCES}

Anders E. \& Ebihara M. 1982, Geochimica et Cosmochimica Acta, 46, 2263

BeppoSAX Cookbook, http://www.sdc.asi.it/software/cookbook/cross.htm]

Boella G. et al., 1997a, A\&AS, 122, 299

Boella G. et al., 1997b, A\&AS, 122, 327

Boyle B.J., Fong R., Shanks T., \& Peterson B.A., 1987, MNRAS, 227, 717

Brinkmann W, Siebert J., Boller T. 1995a, A\&A, 281, 355

Brinkmann W, Siebert J., Reich W., Fuerst E., Reich P., Voges W., Tuemper J., Wielebinski R., 1995b, A\&A S, 109, 147

Cagnoni I. \& Fruscione A. 1994, ApJ, submitted 
Canizares C.R. \& Kruper J., 1984, ApJ, 278, L99

Cappi M., Matsuoka M., Comastri A., Brinkmann W., Elvis M., Palumbo G.G.C., \& Vignali C., 1997, ApJ, 478, 492

Chartas G., et al., 2000, in preparation

Chiappetti L. et al. 1999, ApJ, 521, 552

Cersosimo J.C. et al., 1994, ApJS, 95, 157

Condon J.J., Colton W.D., Greisen E.W., Yin Q.F., Perley R.A., Taylor G.B. \& Broderick J.J. 1998, AJ, 115, 1693

Dermer C.D. \& Schlickeiser R., 1993, ApJ, 416, 458

Dickey J. \& Lockman F.J., 1990, ARAA, 28, 205

Elvis M., Fiore F., Wilkes B.J., McDowell J.C. \& Bechtold J., 1994a, ApJ, 422, 60

Elvis M., Matsuoka M., Siemiginowska A., Fiore F., Mihara T., \& Brinkmann W., 1994b, ApJL, 436, L55

Elvis M. et al., 1994c, ApJS, 95, 1

Elvis M., Fiore, F., Giommi P. \& Padovani P., 1998, ApJ, 492, 91

Fiore F. Elvis M., Giommi P. \& Padovani P., 1998, ApJ, 492, 79

Fiore F., Guainazzi M., Grandi P., 1998, SAX Data Center Technical Report.

Fossati G., Maraschi L., Celotti A., Comastri A., Ghisellini G. 1998, MNRAS, 299, 433

Frontera F. et al., 1997, A\&AS, 112, 357

Fruscione A. et al., 2000, in preparation

Ghisellini G., Fossati G., Maraschi L., Celotti A., Comastri A., 1998, MNRAS 301, 451.

Grandi P. et al., 1997, A\& A, 325, L17

Guainazzi M. \& Matteuzzi A. 1997, BeppoSAX SDC report TR-011, ftp://www.sdc.asi.it/pub/sax/doc/rep tr014.ps.g]

Hamann F. \& Ferland G., 1993, ApJ, 418, 11

Hartman, R.C, et al., 1999, ApJS, in press

Iwasawa K. \& Taniguchi Y., 1993, ApJ 413, L15.

Koenigl A. Kartje J.F., Bowyer. S, Kahn S.M. \& Hwang, C.-Y. 1995 ApJ, 446, 598

Levine A.M. et al., 1984, ApJS, 54, 581

Magdziarz P. \& Zdiarski A.A. 1995, MNRAS, 273, 837 
Marscher A., 1988, ApJ, 334, 552

Marshall F., Boldt E.M., Holt S.S, Miller R.B., Mushotzky R.F., Rose, L.A., Rothschild R.E. \& Serlemitsos P.J. 1980, ApJ, 235, 4

Matt G., 1998, astro-ph/9811053

Mattox, J.R., 1994, BAAS, 185, 8803

Mineo T., F.Fiore, Laor. A, Costantini. E, Comastri A., Elvis M., et al., A\& A, 359, 471.

Morabito D.D., Niell A.E., Preston R.A., Linfield R.P., Wehrle A.E., \& Faulkner J. 1986, AJ, 91, 1038

Moshir N. 1989, 'IRAS Faint Source Survey, Explanatory Supplement version 1, [Pasadena: Infrared Processing and Analysis center] Cal. Tech.

Nandra K. \& Pounds K.A. 1994, MNRAS, 268, 405

Nandra K., Fabian A.C., Brandt W.N., Kunieda, H., Matsuoka M., Mihara T., Ogasaka Y. \& Terashima Y. 1995, MNRAS, 276, 1

O’Dea C.P., 1998, PASP, 110, 493.

Padovani P., Giommi P. 1996, MNRAS, 279, 526

Padovani P., Giommi P. \& Fiore F., 1997, MNRAS, 284, 569

Parmar A. et al., 1997, A\&AS, 112, 309

Quiniento Z. M. \& Cersosimo J.C. 1993, A\&AS, 97, 435

Sakano M., Koyama K., Tsuru T., Awaki H., Ueda Y., Takahashi T., Akiyama M., Ohta K. \& Yamada T., 1998, ApJ, 505, 129

Sambruna, R.M., Maraschi L. \& Urry C.M., 1996, ApJ, 463, 444

Sambruna, R M., George, I. M., Madejski, G., Urry, C. Megan, Turner, T. J., Weaver, K. A., Maraschi, L., Treves, A., 1997, ApJ, 483, 774.

Sambruna, R.M., Eracleous M. \& Mushotzky R.F., 1999, ApJ, 526, 60

SAXDAS software package, release 1.3, Sept. 1997, http://www.sdc.asi.it/software/saxdas

Schartel N., Walter R., Fink H.H., Trümper J. 1996, A\&A, 307, 33

Siebert J., Matsuoka M., Brinkmann W., Cappi M., Mihara T. \& Takahashi T. 1996, A\&A, 307,8

Siemiginowska A. \& Elvis M, 1994, ApJS, 92, 623.

Siemiginowska A., Kuhn O., Elvis M, Fiore F., McDowell J.C. \& Wilkes B.J. 1995, ApJ, 454,77 
Sikora M., Sol H., Begelman M.C. \& Madejski G.M., 1996, MNRAS, 280, 781

Steppe H., Salter C.J., Chini R., Kreysa E., Brumswig W. \& Lobeto Perez J. 1988, A\&AS, 75,317

Turner T.J. et al., 1995, ApJ, 445, 660

Turner E.L. 1991, AJ, 101, 5

Turnshek D.A., 1988, in "Quasar Absorption Lines" eds. J.C. Blades, D. Turnshek \& C. Norman, [CUP: Cambridge], p.17.

Williams O.R. et al., 1992, ApJ, 389, 157

Wilkes B.J., Wright A.E., Jauncey D.L. \& Peterson B.A. 1983, Proc. ASA 5, 2

Wilkes B.J., 1997, private communication

Yaqoob T., George I.M., Nandra K., Turner T.J., Zobair S. \& Serlemitsos P.J., 1998, ApJL, 525, L9

Yaqoob T., George I.M., Turner T.J., Nandra K., Ptak A. \& Serlemitsos P.J., 1999, ApJL, 505, L87

Zenzus J.A., 1997, ARAA, 35, 607 